\title{
Analysis of Clothing Deformation During Motion and its Application for the Design of Functional Clothing
}

\author{
Doudou ZHANG, Sybille KRZYWINSKI, Yordan KYOSEV \\ TU Dresden, Institute of Textile Machinery and High Performance Material Technology, Germany
}

https://doi.org/10.15221/20.09

\begin{abstract}
On the one hand, functional clothing often requires materials with low elongation -for instance conductive wires for signals or antennas, stiff tapes for force transmission or multi-layer membranes with special protective functions. On the other hand, wearing comfort depends on the physical freedom of movement, which can be enhanced by larger gaps in clothing or elastic materials. Hence, this work presents a method for the analysis of clothing deformation during motion and the detection of areas with lower deformation, where wires and tapes can be placed. For the analysis, a human leg with a specially designed elastic textile material was scanned with a 3D scanner in different poses, and subsequently, the 3D data was processed in a two-step procedure: areas with an elongation below a set limit can then be marked and selected for the placement of inelastic functional elements.
\end{abstract}

Keywords: 3D body scanning, skin deformation, clothing deformation, strain field

\section{Introduction}

Mobility is a basic human need and a prerequisite for the integration of people into an inclusive society. With growing life expectancy and the associated demographic change, the topic of mobility is becoming increasingly important. Due to the effort involved, the elderly often avoid leaving their homes without outside help. This reduces social communication in everyday life, which in turn can have a negative impact on their psyche and health. Losing mobility is one of the key risks of aging. While the sports sector has been working on material and pattern cuts development for functional clothing for decades, the customer base of older people has so far been largely neglected. Digital development strategies make it possible to correctly grasp and simulate body shapes and functionalities that change significantly in line with age Modern technology such as 4D scanners help us understand how the human body deforms in motion and how whether clothing promotes wear comfort.

This work was aimed at visualizing the strain field of the textile fabric during human motion by use of information provided by several 3D scans. Based on the strain field, lines of non-extension can be detected, where the body motion causes neither stretching nor contraction. Along these lines, digital components (sensors, microcomputers, and physical components) can be integrated, which will not be negatively affected by body movements of the wearer.

\section{State of the art}

Numerous studies have been carried out in order to analyze skin deformation and develop improved mechanical interfaces with the body. This knowledge can contribute to the design of more comfortable second-skin interfaces for exoskeletal or prosthetic applications or other functional clothing. Current prosthetic liners composed of silicon rubber do not dynamically adapt to changes in posture. Understanding the strain behavior of the targeted body area, including the maximum, minimum, and zero strain fields, is a critical part of designing enhanced interfaces [1]. The first work on skin deformation was introduced by lberall [2]. Based on his observations, lines of non-extension were established, which are notional lines that run across the human body and along which body movements cause neither stretching nor contraction.

Bethke [3] and Marecki [1] further studied skin strain with computational methods for the automated identification of skin strain fields. They used inextensible cabling along the lines of non-extension to provide the required structure for a mechanical counter pressure suit while still allowing full range of motion.

Neumann [4] developed a system for Adidas AG to visualize a complete 3D strain field, which has been actively applied in the industrial context by the company. The strongest deformation of loose-fit as well as tight-fit garments occurs during sporting movements. Choi [5] aimed at identifying the body region 
with the lowest deformation, and therefore, optimal positions for seams, e.g. in terms of functional compression pants.

These works demonstrated several activities in the deformation analysis of human clothing; nevertheless the presented single case studies cannot be applied directly to current research. For this reason, the main ideas of these research projects were re-implemented in order to derive a functional workflow for the strain field analysis of moving clothing.

\section{Data collection and preparation}

\subsection{Subject and poses}

A female subject with a mass of $60 \mathrm{~kg}$ and a height of $165 \mathrm{~cm}$ was used for workflow development. The subject wore a specially designed leggings printed with a square $10 \mathrm{~mm}$ square grid (Fig. 1). This piece of clothing and the resulting images enabled the evaluation of the cloth strain field, based on which the process can be verified; however, this process step hast not yet been completed upon submission of this paper. The leg was captured with a 3D scanner Artec Leo [6]. Fig.1 shows the images obtained from four scans of the subject in four different postures.

In order to analyze the clothing deformation in the leg region during knee bending, postures with different knee flexion angles of $180^{\circ}$ (straight) , $136^{\circ}, 114^{\circ}$, and $97^{\circ}$ (almost sitting) were chosen. These flexion angles were measured based on the angle between femur and tibia bones by means of a goniometer.
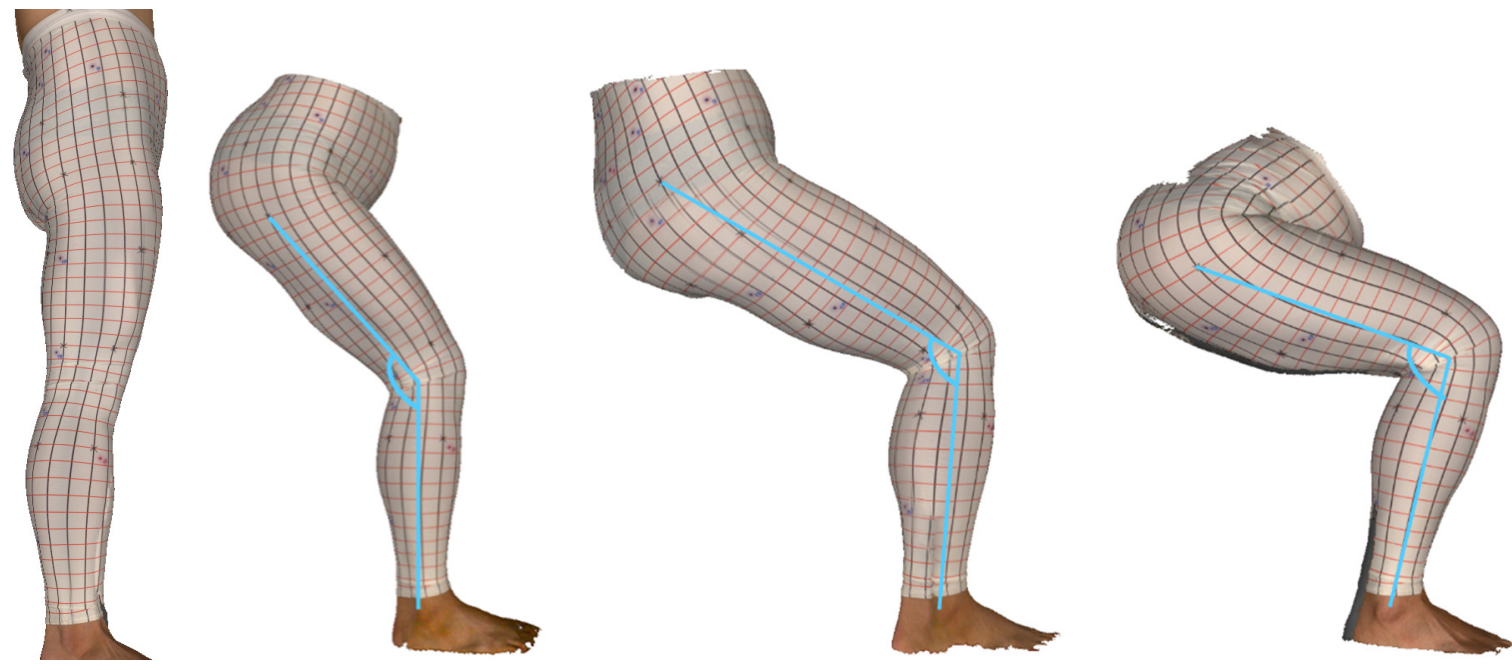

Fig. 1. Screenshots of $3 D$ laser scan models of the leg in various poses (knee flexion angles of $180^{\circ}, 136^{\circ}, 114^{\circ}$, and $97^{\circ}$ ).

During data processing, the right leg was isolated with the help of Artec Software in each scan model. It is important to recognize that the data presented was provided by a pilot study with a single subject and a small number of measurements. Detailed further experiments are necessary in order to determine whether the detected leg shape changes are consistent for a wide sample of body sizes, human types and ages.

\subsection{Problem Size Reduction}

The Artec Scan provided a large number of points with a distance of less than $1 \mathrm{~mm}$ between them. Scan data was remeshed with the Software InstantMeshes [7] in order to obtain a low-resolution triangle mesh. The number of triangles was reduced from 459410 (Figure 2a) to 809 (Figure 2b), which is approx. 500 times less than the original scan so that subsequent data processing is 500 times faster without a significant loss of accuracy in the current study. If, in the case of other studies, the mesh size is not accurate enough or the volume representation is not smooth enough, the mesh size can be flexibly adjusted; thus, the following computational steps can be used in the same way. 


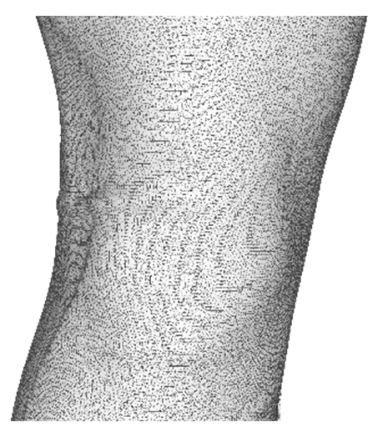

a

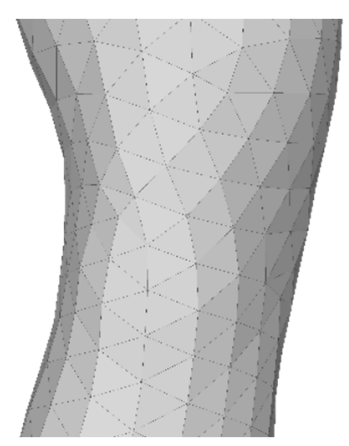

b

Fig.2. Reduction of triangle number, a) high-resolution scan; b) low-resolution triangle mesh.

\subsection{Correspondence problem}

Each 3D scan consists of a set of point clouds and triangle patches for each pose, however, there is a lack of correspondence between them, i.e. vertex number $\mathrm{N}$ of pose 1 does not correspond to the same position as vertex number $\mathrm{N}$ of pose 2 . In order to solve this correspondence issue, the point clouds of each body pose must be equally structured and identical triangulation must be used for the strain metrics to be computed properly. The correct correspondence between points and triangles of different body configurations is critical for computational accuracy. In our research, the described correspondence problem, i.e. the automatic matching between two data sets, was solved with the help of the software Wrap3 [8]. Using a feature termed "wrapping", the fitting process can be controlled by providing self-defined point correspondences. "Wrapping" is a very efficient approach when dealing with extensive sets of similar objects, such as human scans, so that the same topology is applied to all of them. Figure 3 shows the calculated results with mesh topologies being identical for all poses of the same subject. This is a very important prerequisite for strain analysis on each triangle based on comparisons between different knee flexion angles.

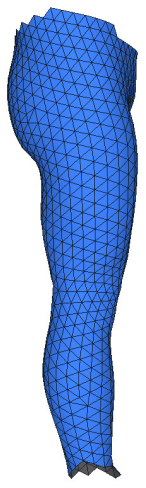

a

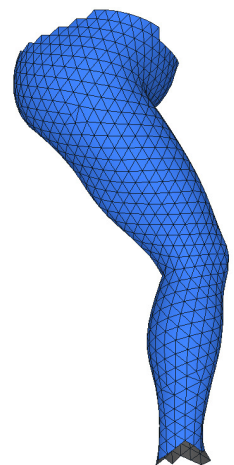

b

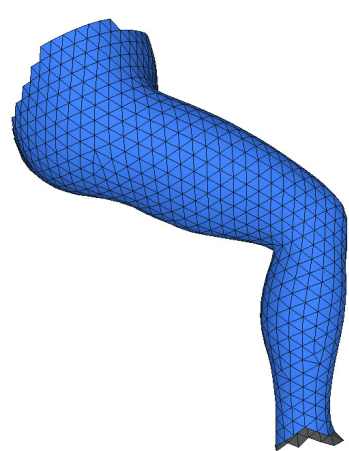

C

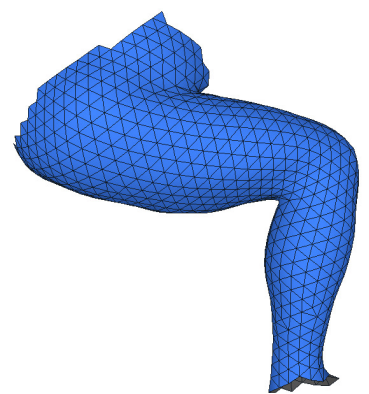

d

Fig.3. Triangle meshes generated with the identical topologies

\section{Analysis method - constant strain triangles}

As opposed to metals, wood and other solid materials, textile materials are subject to large deformations during wearing. The numerical strain computation of deformed materials depends on the selection of the reference coordinate system and the type of linearization of strains. The work presented in this paper followed an algorithm according to Marecki [1]. The change of position and form of the triangle between two time steps $\mathrm{t}=0$ and $\mathrm{t}=1$ was considered. The triangle element (patch) at $\mathrm{t}=0$ had the coordinates $\left\langle p_{1}^{0}, p_{2}^{0}, p_{3}^{0}\right\rangle$ and $\left\langle p_{1}^{1}, p_{2}^{1}, p_{3}^{1}\right\rangle$ after a certain time period, respectively; for each point $p_{i}^{k}$, the subscript $i$ denotes the index number of the edge of the triangle, whereas the supper-script $k$ refers to the time (Figure 4). 

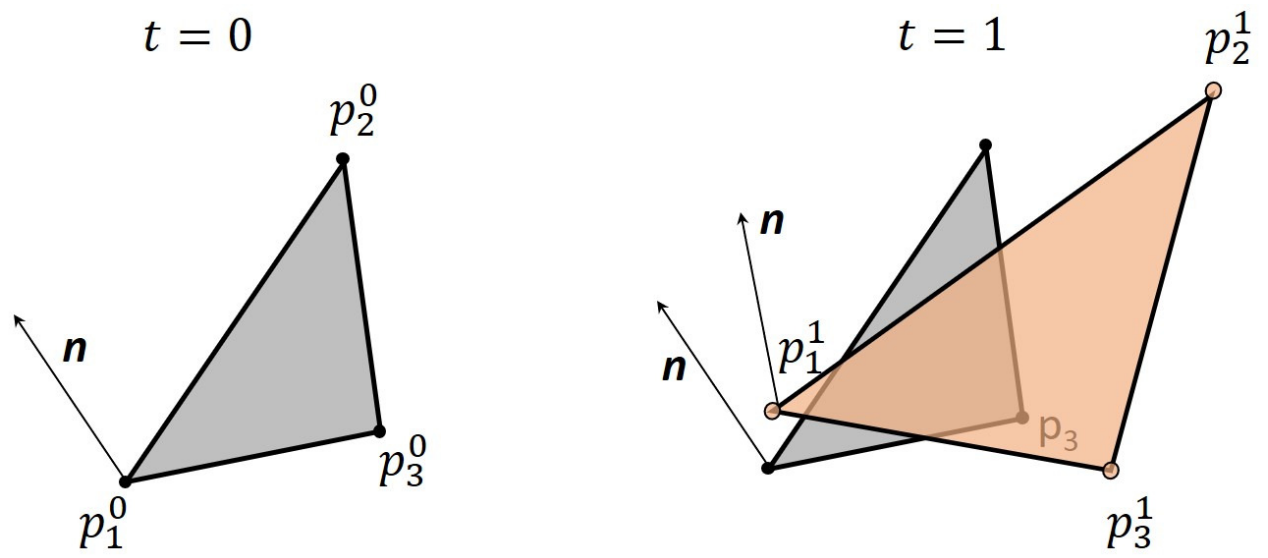

Fig. 4. Deformation of a single triangle element

Due to large displacements and rotations, the standard linear elasticity theory could not be directly applied for the strain state calculations of this triangle. Figure 5 represents an idealized version of the decomposition and deformation steps occurring between two states. Each patch is translated to its new position (translation $\mathrm{T}$ ) and rotated into the new orientation (rotation $\mathrm{R}$ ); also, extension in both directions ( $\mathrm{S}$ as scaling) and shear $(\Theta$, recognized as a change in angles between the patch sides) can be applied.
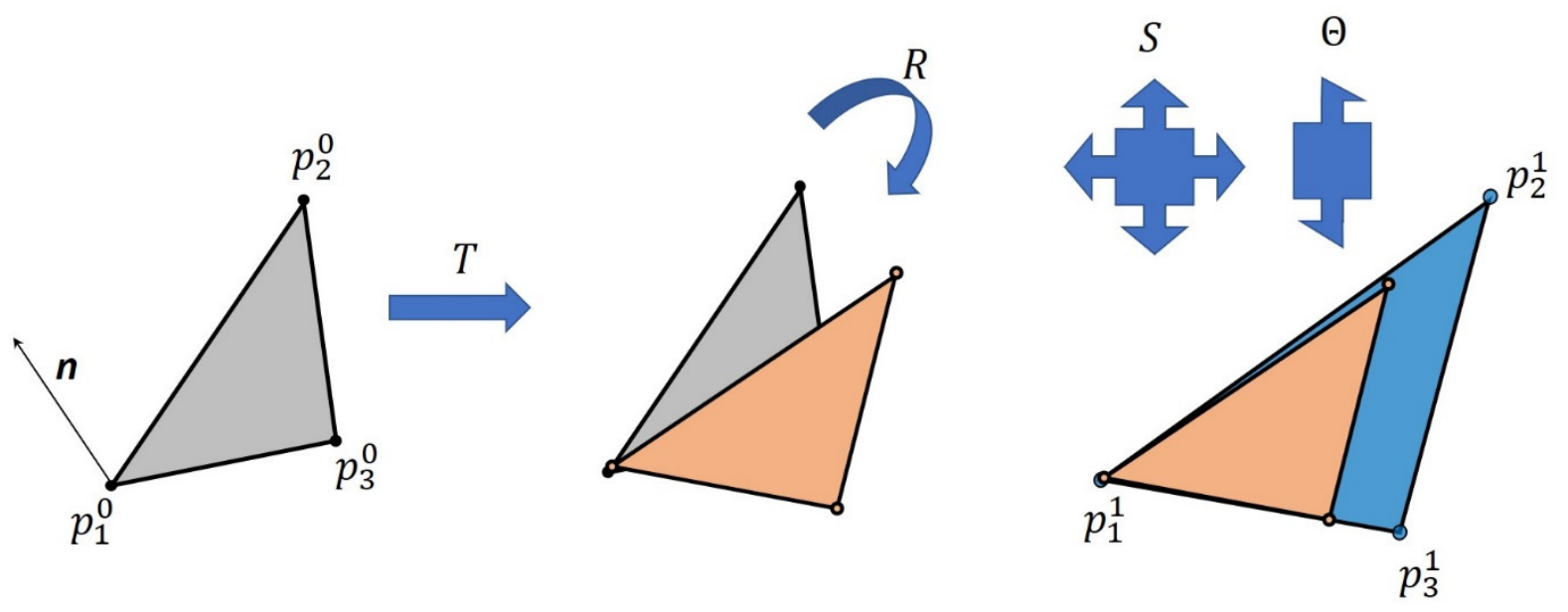

Fig. 5. Decomposition of motions and deformations.

By means of singular value decomposition of the transformation matrix, the single rotations and scaling in both unique vector directions can be extracted, and finally, the strain of the element can be calculated. At the current state, the von Mises strain or an equivalent strain can be calculated based on the following equation

$$
\varepsilon_{\text {rel }}=\frac{1}{2} \sqrt{\left(\varepsilon_{1}-\varepsilon_{2}\right)^{2}+\varepsilon_{1}^{2}+\varepsilon_{2}^{2}}
$$

where $\varepsilon_{1}$ and $\varepsilon_{2}$ are the relative strains in the patch between initial and current poses.

\section{Results}

Figure 6 shows the strain plot of a human leg in flexion. This leg is elastic and was prepared to be smaller than the real leg size, i.e. all areas were pre-deformed by applying initial elongation. Thus, the calculated strain between the initial pose and the following poses represents the relative strain $\varepsilon_{\text {rel }}(i)$ of the structure. The determination of the full absolute strain of the structure requires the knowledge of the initial state at each point $i$ of the leg $\varepsilon_{0}(i)$. The absolute strain $\varepsilon_{a b s}(i)$ can then be obtained based on

$$
\varepsilon_{a b s}(i)=\varepsilon_{r e l}(i)-\varepsilon_{0}(i)
$$


Its determination requires the measurement (manually or automatically, using image processing) of the current length of each cell of the mesh as well as the assignment of initial strains to the corresponding triangle patches. This procedure has not yet been performed since the calculation of relative strain is sufficient for the evaluation of textile deformations. For this reason, the research presented in this paper focused on relative strain analysis. The detection of negative relative strains did not necessarily imply folding of the textile material. In these areas, the material relaxes initially: folding can start if the absolute strain becomes negative.

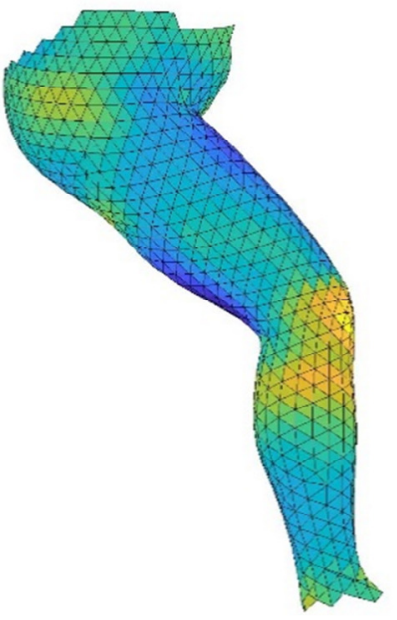

a

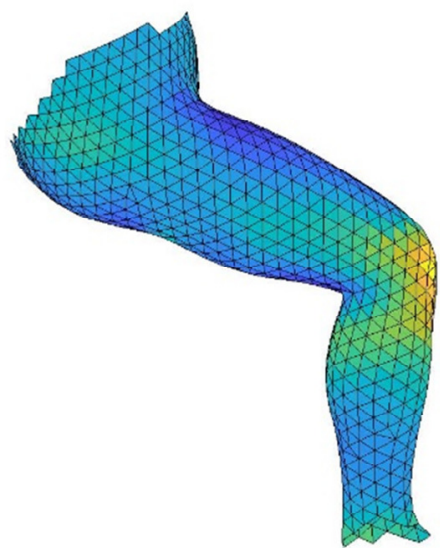

$b$

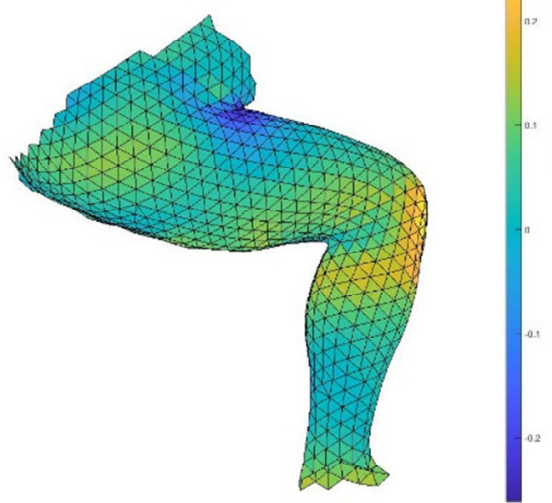

$c$

Fig. 6. Equivalent relative strains in the patches after motion a) step 1, b) step 2, c) step 3

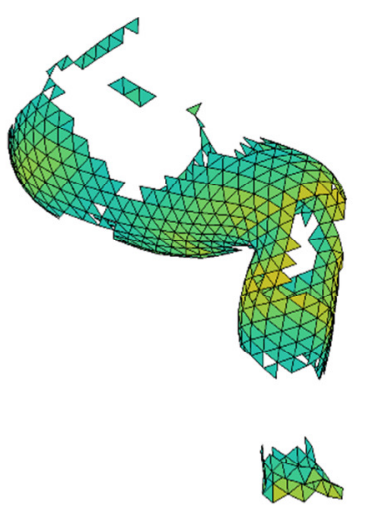

a

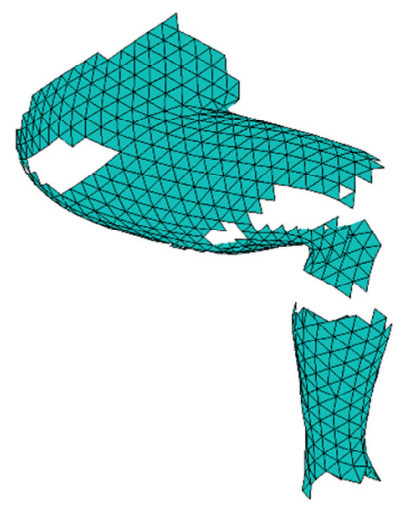

$b$
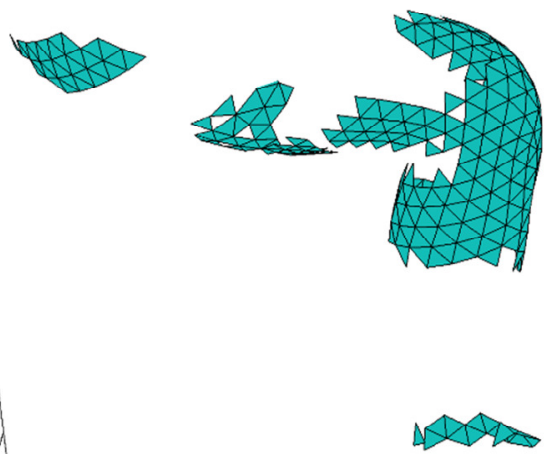

$c$

Figure 7. Selected areas with different equivalent strains a) equivalent strain (related to the initial state) in the range of $-0.1<\varepsilon<0.1$ b) areas with negative relative strain (compression or relaxation) in pose four c) areas with additional tensile loading - positive relative strain

Figure 6 demonstrates the relative strains for the poses 2, 3 and 4. As expected, there are areas with larger relative extension and areas where relaxation occurred (negative relative strain). Although it was anticipated that a higher knee angle would lead to a larger extension in the upper knee area, this behavior could not be observed. This is most likely due to the relative motion (slippage) of the leggings over the leg compensating the increasing length of the contact area.

Figure $7 \mathrm{a}$ visualizes areas with a relative strain of less than 0.1 in both the tension and relaxation direction. These areas are suitable for the placement of cables and other rigid elements (plates, LED), which require less elongation. The elongation limit of 0.1 was selected as example and can be adjusted as a parameter. Figure $7 \mathrm{~b}$ visualizes the areas where the strain is negative, which means that patches underwent relaxation (compression) and became shorter than the first scanned state.

Areas with positive strain, shown in Figure 7c, exhibited a larger elongation compared to their initial state. They are unsuitable for conductive cables and other non-elastic connectors because they will not be able to follow material deformations, leading to higher forces acting on adjacent joints. 
Figure 8 depicts all strains of the triangles in all three states, sorted according to ascending strains. The triangle order is not identical for all three diagrams since during motion load redistribution was detected, thus requiring a more detailed analysis. However, this is a task yet to be completed. Contrary to initial expectations, step 3 demonstrates more triangles with a positive strain, while reaching the same tensile strain values in maximum loaded areas. This effect also became visible in Figure 6. A potential explanation for this result involve the areas underneath the knee getting significantly "compressed" or relaxed during bending, which leads to an increased number of patches with a negative strain. The question as to why the strain at the upper knee is relatively low can be explained by the redistribution of material in terms of the total length of the garment.

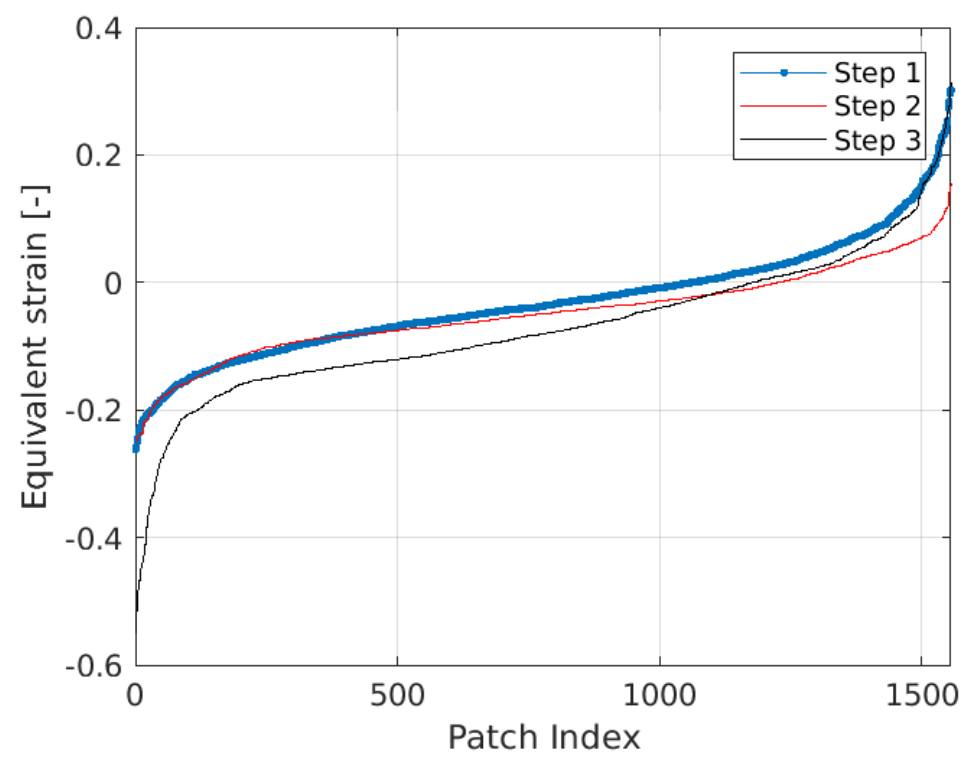

Figure 8. Strain of the triangles (patches) in all three states, sorted ascending

\section{Discussions}

The analysis of these first results demonstrated that the deformation of clothing is a complex process. The proper interpretation of results requires in-depth knowledge of material behavior - for instance the maximal possible elongation of material at loads typically occurring during human motion and the initial strain of the material after it was placed on the body. If these values are provided, areas where wrinkle formation starts and maximum strain is achieved can be identified. During leg motion, the material slides on the human body surface, which leads to the redistribution of the deformation. As a result, textile deformations are different from deformations of human skin. In order to analyze them, the displacements of triangles must also be considered. To investigate the full range of behavior, data including the initial deformation of triangles is required, which must then be analyzed and assigned to corresponding triangles; however, these research efforts require further algorithm developments and are subject to future projects.

\section{Conclusion}

A workflow for the analysis and visualization of relative strain of a textile material during human motion was implemented and tested for four leg poses of a female subject. The developed triangle-based strain analysis enables the efficient and repeatable visualization of the strain field. However, further algorithms must be developed and arranged to corresponding triangles for the determination of absolute strains. Nevertheless, the current algorithm allows for the strain field analysis of tight-fitting textiles, based on which suitable areas for the placement of inelastic components of functional clothing and general deformation patterns can be identified. 


\section{References}

[1] A. Marecki, „Skin strain analysis software for the study of human skin deformation“, 2012.

[2] A. S. Iberall, The Use of Lines of Nonextension to Improve Mobility in Full-pressure Suits. Aerospace Medical Research Laboratories, 1964.

[3] K. A. Bethke, The second skin approach : skin strain field analysis and mechanical counter pressure prototyping for advanced spacesuit design. Thesis, Massachusetts Institute of Technology, 2005.

[4] T. Neumann, „Reconstruction, Analysis, and Editing of dynamically deforming 3D-Surfaces“, 2016, Zugegriffen: Sep. 11, 2019. [Online]. Verfügbar unter: https://publikationsserver.tubraunschweig.de/receive/dbbs_mods_00064146.

[5] J. Choi und K. Hong, „3D skin length deformation of lower body during knee joint flexion for the practical application of functional sportswear", Applied Ergonomics, Bd. 48, S. 186-201, Mai 2015, doi: 10.1016/j.apergo.2014.11.016.

[6] „Wireless Handheld 3D Scanner | Artec Leo | Best 3D Scanning Solutions“, Professional 3D scanning solutions / Artec3D. https://www.artec3d.com/portable-3d-scanners/artec-leo-v2 (zugegriffen Aug. 17, 2020).

[7] „igl | Interactive Geometry Lab | ETH Zurich | Instant Field-Aligned Meshes“. https://igl.ethz.ch/projects/instant-meshes/ (zugegriffen Aug. 28, 2020). 